JHR

32,3

198

Received 6 September 2017 Accepted 23 January 2018

\section{Influence of emotional regulation on successful aging among Thai older adults}

\author{
Arunya Tuicomepee, Panrapee Suttiwan, Rewadee Watakakosol and \\ Sakkaphat T. Ngamake \\ Faculty of Psychology, Chulalongkorn University, Bangkok, Thailand, and \\ Sompoch Iamsupasit \\ College of Public Health Science, Chulalongkorn University, Bangkok, Thailand
}

\begin{abstract}
Purpose - Successful aging represents a positive development in older adults. The emphasis on aging well has sought to understand resources such as emotional regulation that facilitates healthy and happy aging. The purpose of this paper is to investigate the impact of two common emotional regulation strategies (i.e. cognitive reappraisal and expressive suppression) on successful aging among Thai older adults.

Design/methodology/approach - The participants selected for this paper were 150 older adults living in Bangkok and adjacent areas. Their mean age was $69.7( \pm 6.7)$ years old. Instruments were the Successful Aging Inventory and Emotion Regulation Questionnaire. Each participant voluntarily completed an individual self -reported questionnaire.

Findings - The results revealed that the two emotional regulation strategies (cognitive reappraisal and expressive suppression) have collectively explained 6 percent of the variance of successful aging. The cognitive reappraisal strategy was a sole significant predictor $(\beta=0.20)$.

Originality/value - Promoting emotional regulation strategies in particular a cognitive reappraisal strategy among Thai older adults can facilitate their cognitive functioning, and their successful aging.
\end{abstract}

Keywords Emotional regulation, Successful ageing, Thai older adults

Paper type Research paper

\section{Introduction}

Healthy and successful aging is possibly the most important research outcome and policy decisions on aging and the aging population. Rowe and Kahn[1] were the first to propose the concept of successful aging. Their work through the MacArthur Foundation and other research networks generated research on aging. In Thailand, a qualitative investigation revealed that successful aging of Thai older adults defined as "the elderly with good physical and mental health, with enthusiasm and physical flexibility, with ability to live within the family and the society happily, with delight and satisfaction from the pride in their own past occupational success or the present success of their descendants"[2,3].

These successful aging characteristics are supported by the Rowe and Kahn[1] concept of successful aging, which focuses on such primary factors as less susceptibility to disease and impairment, the ability to function mentally and physically, harmonious participation in society, and passion for life[1]. An empirical study on Thai successful aging by

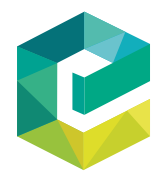

(C) Arunya Tuicomepee, Panrapee Suttiwan, Rewadee Watakakosol, Sakkaphat T. Ngamake and Sompoch Iamsupasit. Published in the Journal of Health Research. Published by Emerald Publishing Limited. This article is published under the Creative Commons Attribution (CC BY 4.0) licence. Anyone may reproduce, distribute, translate and create derivative works of this article (for both commercial \& noncommercial purposes), subject to full attribution to the original publication and authors. The full terms of this licence may be seen at http://creativecommons.org/licences/by/4.0/legalcode

The project was supported by a research grant from the 2015 Chulalongkorn University's Research Development on Cluster of Aging Society Fund, Chulalongkorn University, Thailand.
Journal of Health Research pp. $198-202$ Emerald Publishing Limited 2586-940X DOI 10.1108/JHR-05-2018-02 Vol. 32 No. 3, 2018 
Wattagakosol et al.[4] revealed that successful aging included multiple components involving psychological factors such as mindfulness, family functioning, and social engagement. They defined successful aging as five interrelated dimensions (i.e. body, mind and emotion, brain and cognition, social, and life wisdom). The body dimension refers to successful physical aging or health conditions. An example of index on this dimension is as "my health conditions are better than those of people in my age group." The mind and emotion dimension refers to successful emotional aging. An example of this dimension is "I am always disappointed in my life." The brain and cognition dimension refers to successful cognitive aging. An example of this dimension is "I could pass on experience and knowledge to the next generations." The social dimension refers to successful social aging. An example of this dimension is "I am an important person to my family and close friends." These dimensions were significantly and positively related to a hypothetical construct of successful aging, with social engagement having the strongest relationship, followed by family functioning and mindfulness, respectively[4].

A previous literature review has identified the contributing factors to successful aging such as emotional regulation as ways of coping with normative stressors among older adults[5-7]. Carstensen[8], a Theorist on aging, proposed the socio-emotional selectivity theory explaining that increasing age prompts a greater emphasis on emotion regulation. This idea has subsequently been elaborated with the suggestion that age-related changes in motivation require greater allocation of resources and more effective strategies to regulate emotion[8]. Emotional regulation is the internal and transactional processes through which individuals consciously or unconsciously modulate one or more components of emotion, by modifying either their own experience, behavioral expression, or the emotion-eliciting situation[5, 6]. So far, two common emotional regulation strategies studied by psychologists and scholars are cognitive reappraisal and expressive suppression. Cognitive reappraisal refers to emotional regulation strategy: individuals change their emotional response by reinterpreting or reframing the meaning of the emotion-eliciting situation. This strategy usually helps individuals to reduce a negative impact of spontaneous emotional state. Another strategy, expressive suppression, refers to the strategy that individuals attempt to hide, inhibit, or reduce ongoing emotion-expressive behavior[6]. Research studies have found that, compared to expressive suppression, cognitive reappraisal leads to a less negative emotional outcome[5-7]. Hence, this current paper explored the role of these two emotional regulation strategies - cognitive reappraisal and expressive suppression - on predicting successful aging among Thai older adults.

\section{Methods}

This study is part of the project entitled "Influence of emotional regulation on subjective well-being and successful aging in Thai elderly and their caretakers: a dyadic modeling study." The study was approved by the Ethical Review Committee for Research Involving Human Research Subjects, Health Science Group, Chulalongkorn University. Details of research method are as below.

\section{Participants}

Healthy individuals at least 60 years old who live in an urban area of Bangkok were recruited in this study. They were excluded if there is a significant memory problem. In total, 150 older adults with mean age of $69.7(\mathrm{SD}=6.7)$ years participated in this study. Of the participants, four-fifths (80 percent) were female. Approximately one-third (37.3 percent) were married. 
JHR

32,3

200

\section{Instrument}

A survey packet consists of items asking about demographic information (i.e. gender, age, marital status) and two psychological measures, one developed and validated particularly for use with Thai population: the Successful Aging Inventory, and another translated from a well-validated and commonly used measure: the Emotional Regulation Questionnaire.

The revised Successful Aging Inventory (SAI-R). This revised inventory was developed by a researcher team. The original SAI contained five core dimensions of successful aging: body, mind and emotion, brain and cognition, social, and life wisdom. However, life wisdom dimension was dropped eventually due to a lack of conceptual precision and poorly defined psychometric properties[4]. This study used a revised version of the Successful Aging Inventory, which contains 20 items measuring mental, physical, and social functioning of the elderly in four dimensions: body, mind and emotion, brain and cognition, and social. Responses were rated on a three-point rating scale, ranging from $1=$ not true to me to $3=$ true to me. Relatively, high scores indicate good standing in terms of global successful aging or its dimensions. For this study, total successful aging scores were served as criteria in the regression models. The scale in this study demonstrated good level of internal consistency (Cronbach's $\alpha=0.76$ ).

Emotion Regulation Questionnaire (ERQ-T). This study used the Thai version of the ERQ-T. The questionnaire was translated from the original version developed by Gross and John[5]. It includes ten items assessing the tendency to use two emotion regulation strategies: cognitive reappraisal and expressive suppression. The participants were asked to answer to each item on a seven-point Likert-type scale, ranging from $1=$ strongly disagree to $7=$ strongly agree. Higher scores on each strategy reflect more usage of that particular strategy. The subscales of ERQ-T in this study demonstrated a good level of internal consistency ( $\alpha=0.74$ for the cognitive reappraisal subscale and $\alpha=0.60$ for the expressive suppression subscale).

\section{Data collection}

Trained research assistants approached people aged over 60 in various community settings located in a rural area of Bangkok. As suggested by Kelley and Maxwell[9], sample size approximation of one 100 for multiple linear regressions is adequate with three predictor variables and expects $R^{2}$ population to be about 0.02 . This study, 150 participants were recruited for precise estimates of standardized regression coefficients. They were selected using convenient sampling. Prospective participants were invited to participate in a research study regarding emotion regulation and mental health among elderly people. Once informed of the purpose and importance of the study, as well as the confidentiality and the anonymity of the data, the participants signed a consent form and then responded to a questionnaire packet. They could read and give written responses to the questionnaire packet themselves, or have a research assistant read aloud and mark responses for them. They were then thanked and given a small gift. No adverse consequence was observed during the data collection period, and a contact to a psychological service center (i.e. hotline) was provided those who felt uneasy after participating in the study.

\section{Data analysis}

Means, standard deviations, Pearson product moment correlation coefficients, and multiple linear regressions among successful aging and emotion regulation strategies were used to analyze the data. 


\section{Results}

Table I shows the means, standard deviations, and zero-order correlation coefficients between successful aging and emotion regulation strategies. At a glance, successful aging and its four components were all positively correlated while a substantive correlation coefficient $(r=0.50)$ was observed between the two emotion regulation strategies. Elderly people who reported a high score in one dimension of successful aging tended to report high scores in the other dimensions. Similarly, those who reported relatively frequent use of one strategy to regulate their emotion tended to report using another strategy more often.

To examine the predicted relationships of successful aging and emotion regulation strategies (i.e. cognitive reappraisal and suppression), multiple linear regression analysis was performed. Table II displays regression coefficients, standardized regression coefficients, and coefficients of determination of the variables. In the model predicting successful aging, both predictors have collectively explained only 6 percent of the variance of the criterion variable with the cognitive reappraisal strategy was a sole predictor. Using such a strategy in order to increase positive emotions and decrease negative emotions would be positively associated with the levels of successful aging reported by the elderly.

\section{Discussion}

The findings revealed that the elderly who frequently suppressed their emotional expression (regardless of the valence, be it positive or negative) reported relatively low levels of emotional well-being. On the other hand, the elderly who frequently changed perspectives on many things in their lives to enhance positive emotion, and/or to reduce negative emotion, reported relatively high levels of brain health and cognitive functioning. Previous studies have showed that the way one thinks can affect one's emotional experiences and responses[6]. In this study, older adults who experience and respond to their emotions with the cognitive reappraisal strategy are likely to report high levels of overall successful aging. The role of this strategy was prominent when predicting successful aging. Consistent with the literature, the use of the cognitive reappraisal strategy played important roles in facilitating successful aging by reducing stress exposure in late life, by enhancing social support and other coping resources, and by promoting the use of both preventive and proactive adaptation strategies by older adults $[5,7,8]$.

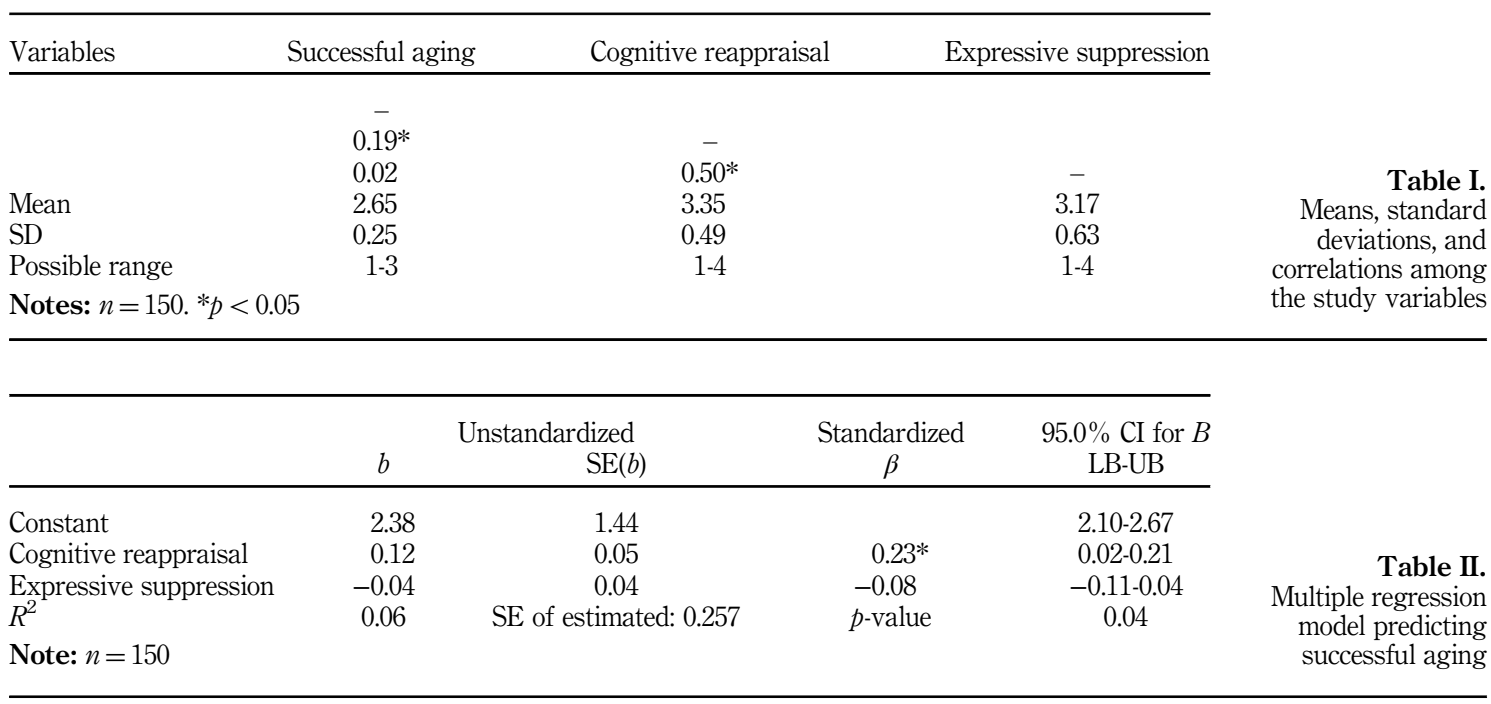


JHR

32,3

Although the findings from this study clearly indicate that successful aging uses both cognitive reappraisal and emotional expression strategies. In line of past research in Western cultures, older adults in this study reported some negative consequences (i.e. reduced emotional well-being) of using the expressive suppression strategy[6]. This may be because Thai older adults, like those in many Asian countries, tend not to express their emotions with the aim of balancing their minds[10]. In the Thai culture, older adults are revered by their family and community. Most of them are surrounded by love and live close to their family. Their expressive suppression strategy may enable Thai older adults to accept the status quo and live in contentment. However, Thai older adults may not actively suppress their emotions (e.g. control/ change their facial expression) because they tend to practice Buddhist principles of "humbleness, equanimity, calmness, acceptance, and tranquility" to nurture the interpersonal harmony[11].

\section{Conclusion}

This study has led to a new understanding of the relationship between emotion regulation strategies and successful aging in elderly Thais. These older adults who used the cognitive appraisal strategy to manage their emotions were more likely to report overall successful aging. In addition, the older adults who frequently suppressed their emotional expression reported less successful aging. The finding has shown that the cognitive reappraisal strategy can facilitate successful aging among older adults. It is therefore important to encourage Thai elders to practice this cognitive appraisal strategy.

\section{References}

1. Rowe JW, Kahn RL. Human aging: usual and successful. Science. 1987; 237(4811): 143-9.

2. Thanakwang K, Soonthorndhada K, Mongkolprasoet J. Perspectives on healthy aging among Thai elderly: a qualitative study. Nurs Health Sci. 2012; 14(4): 472-9.

3. Thiamwong L, McManus MS, Suwanno J. Development of the Thai healthy aging model: a grounded theory study. Nurs Health Sci. 2013; 15(2): 256-61.

4. Watakakosol R, Ngamake S, Suttiwan P, Tuicomepee A, Lawpoonpat C, Iamsupasit S. Factors related to successful aging in Thai elderly: a preliminary study. J Health Res. 2013; 27(1): 51-6.

5. Gross JJ, John X. Individual differences in two emotion regulation processes: implications for affect, relationships, and well-being. J Pers Soc Psychol. 2003; 85(2): 348-62.

6. Gross JJ. Handbook of emotion regulation. 2007; The Guildford Press, New York, NY.

7. Matsumoto D, Yoo SH and Nakagawa S. Culture, emotion regulation, and adjustment. J Pers Soc Psychol. 2008; 94(6): 925-37.

8. Carstensen LL, Gross J, Fung H. The social context of emotional experience. In: Lawton MP, Schaie KW editors. Annual review of geriatrics and gerontology. New York: Springer; 1997; 325-52.

9. Kelley K, Maxwell ES. Sample size for multiple regression: obtaining regression coefficients that are accurate, not simply significant. Psychol Methods. 2003; 8(3): 305-21.

10. Wei M, Su JC, Carrera S, Shu-Ping L. Suppression and interpersonal harmony: a cross-cultural comparison between Chinese and European Americans. J Couns Psychol. 2013; 60(4): 625-33.

11. Tori C, Bilmes M. Multiculturalism and psychoanalytic psychology: the validation of a defense mechanisms measure in an Asian population. Psychoanal Psychol. 2002; 19(4): 701-21.

\section{Corresponding author}

Arunya Tuicomepee can be contacted at: atuicomepee@gmail.com

For instructions on how to order reprints of this article, please visit our website:

www.emeraldgrouppublishing.com/licensing/reprints.htm

Or contact us for further details: permissions@emeraldinsight.com 